\title{
BARRIERS RELATED TO MAMMOGRAPHY IN THE RIVERSIDE POPULATION OF THE BRAZILIAN AMAZON: CASE-CONTROL STUDY
}

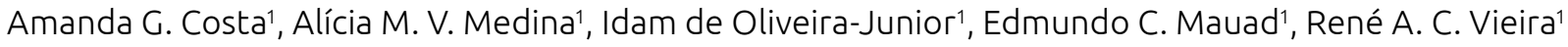 \\ 'Barretos Cancer Hospital, School of Medicine, Faculdade de Ciências da Saúde de Barretos Dr. Paulo Prata - Barretos (SP), Brazil.
}

Background: Mammographic screening promotes early detection in the asymptomatic phase. Due to economic and logistical limitations in Brazil, mammographic screening is not available to all individuals, which is expressed in the high number of patients diagnosed at an elevated stage. There is a lack of knowledge related to the real barriers regarding the challenges to perform mammography in the riverside rural population in Amazônia. Objective: To evaluate the factors related to the limitation of access, knowledge and information on breast cancer of the riverside population in Amazonia. Methods: Ethics Committee approval (1401/2017). A prospective, transversal, case-control study carried out in the urban and riverside area of the city of Porto Velho/Rondônia, in women aged 40-69 years. A mammography examination was offered, and a questionnaire was carried out, evaluating factors related to non-mammography adherence. Descriptive statistics were performed. To compare the groups, the chi-square and the Mann-Whitney test were performed. Results: The convenience sample consisted of 112 women who participated in the study, being 46 of riverside population (case), in a ratio of 1:1.4. Reported reasons for non-compliance were absence of symptoms, difficulty in performing the test, nonmedical request, fear of pain, lack of knowledge of the age of the test. Comparing the characteristics of each group, these did not differ in relation to race $(\mathrm{p}=1.00)$, age $(\mathrm{p}=0.17)$. Evaluating health care, patients in the riverside region had a higher frequency of never having undergone MMG examination in their lifetime $(\mathrm{p}=0.04)$, lack of knowledge about the regularity of MMG ( $\mathrm{p}=0.01)$. Conclusions: Patients in the riverside population presented a higher social vulnerability, associated with a lower rate of MMG testing in life, but had similar knowledge about self-examination, presenting regular medical guidance to MMG. The Mobile Prevention Unit minimized the differences between the groups. 